\title{
El paisaje social del centro de Argentina durante la transición Pleistoceno-Holoceno (ca. 1100o- $9000 \mathrm{AP}$ )
}

\section{Diego E. Rivero* y Guillermo Heider**}

\section{Resumen}

Desde la perspectiva del Análisis de Redes y del Aprendizaje del Paisaje, se evalúa el registro arqueológico correspondiente a la transición Pleistoceno-Holoceno del centro de Argentina (ca. 11000 - 9000 AP). Para ello, se analiza la circulación de materias primas líticas utilizadas para la elaboración de instrumentos, así como el uso de los recursos simbólicos organizados por medio del estilo de las puntas de proyectil. Se considera que las interrelaciones sociales poseen una importancia vital para el mantenimiento de la continuidad social y el éxito de los procesos de colonización inicial de una determinada región. En toda el área de estudio, el cuarzo es la materia prima lítica de mayor disponibilidad; sin embargo, se destaca el uso de rocas silíceas para las puntas de proyectil y algunos otros artefactos. Fuentes potenciales de estas rocas fueron identificadas en las Sierras Centrales (provincias de Córdoba y San Luis). En base a estas evidencias, y al modelo de yacencia de rocas para la región, se discuten las implicancias del uso y circulación de las materias primas líticas para definir el modo de vida y el paisaje social de los primeros pobladores durante la colonización inicial del sector central de Argentina.

\section{The social landscape of central Argentina during the Pleistocene- Holocene transition (ca. 11,000-9000 BP)}

\footnotetext{
Abstract

The archaeological record corresponding to the Pleistocene-Holocene Transition in Central Argentina ( $c a .11,000$ - 9000 BP) is evaluated from the perspective of Network Analysis and Landscape Learning, to understand the Social Landscape in which the individuals interacted with other groups that were socially and/or spatially apart. We analyze the circulation of lithic raw materials used for manufacturing tools, as well

* Instituto de Estudios Históricos (IEH), Centro de Estudios Históricos “Carlos Segreti” (CEH) - CONICET, Universidad Nacional de Córdoba. Raúl Casariego 4096 (CP 5008), Córdoba, Argentina. E-mail: ayampitin1@yahoo.com.ar

** Departamento de Geología, Facultad de Ciencias Físico-Matemáticas y Naturales, Universidad Nacional de San Luís (UNSL). Av. del Fundador 1140, casa 3, (CP 5700), San Luís, Argentina. E-mail: guillermoheider@hotmail.com
}

Recibido: 7 de marzo de 2019

Aceptado:

4 de julio de 2019

\section{Palabras clave}

Tardiglacial

Redes de interacción

Aprendizaje del paisaje
Keywords

Tardiglacial Interaction networks Landscape learning 
as the use of symbolic resources organized according to style in projectile points. We consider that social relationships have a major importance in maintaining social continuity and in the success of the initial colonization processes of a certain region. Quartz is the most abundant raw material in the study area, but siliceous rocks were preferred for the manufacture of projectile points and some other artifacts. Potential sources of these rocks were located in the Central Mountains (Provinces of Cordoba and San Luis). The implications of lithic raw material use and circulation are used to discuss the way of life and the social landscape of the first settlers during the initial colonization of central Argentina.

\section{Introducción}

El objetivo de este trabajo es avanzar en la definición del paisaje social correspondiente a la transición Pleistoceno-Holoceno del centro de Argentina (ca. 11000 - 9000 AP) a partir del uso y circulación de materias primas líticas. Para ello se utilizan las herramientas teóricas del aprendizaje del paisaje (Rockman, 2003, 2009) y del análisis de redes (Gamble, 2001). En este sentido, se analiza la circulación de materias primas líticas utilizadas para la elaboración de instrumentos, así como el uso de los recursos simbólicos organizados por medio del estilo en algunos de estos artefactos formatizados, como las puntas de proyectil.

Las investigaciones desarrolladas en el área central de Argentina consiguieron, durante los últimos años, determinar aspectos significativos de la estructura del registro arqueológico atribuible a los primeros grupos humanos que ocuparon la región. El descubrimiento de contextos arqueológicos tempranos y el incremento de dataciones absolutas permitieron confirmar la presencia humana durante la transición PleistocenoHoloceno (ca. 11000 - 9000 AP), así como avanzar sobre la comprensión de los modos de vida durante el poblamiento inicial (Rivero, 2009, 2012; Rivero y Berberián, 2006, 2008; Rivero y Roldán, 2005; Roldán, Rivero y Pastor, 2005).

Los estudios de Ameghino (1885) y Castellanos (1943) fueron pioneros en postular gran antigüedad para la presencia humana, aún en un contexto donde primaban las ideas que sostenían que la misma era acotada (Serrano, 1945; Vignati, 1940). Sin embargo, recién en la segunda mitad del siglo XX se pudieron constatar ocupaciones tempranas de cazadores-recolectores en ca. 8000 años AP (González, 1960). La posibilidad de una mayor profundidad temporal, si bien fue planteada por algunos indicios indirectos, no pudo ser demostrada hasta las investigaciones efectuadas en el sitio El Alto 3 (en lo sucesivo EA3), localizado en el sector central de las Sierras Grandes de Córdoba (Figura 1). En esa localidad arqueológica se obtuvieron tres fechados radiocarbónicos asociados a material arqueológico en el componente inferior (C1A), que dieron una antigüedad de $9371 \pm 51$ años $\mathrm{C}^{14} \mathrm{AP}$ (AA94987; madera carbonizada), $9790 \pm 80$ años $\mathrm{C}^{14} \mathrm{AP}$ (LP-1420; madera carbonizada) y $11010 \pm 80$ años $\mathrm{C}^{14} \mathrm{AP}$ (LP-1506; madera carbonizada) respectivamente (Rivero, 2009, 2012).

Por otra parte, se logró datar un fragmento de cráneo en $10450 \pm 50$ años $C^{14} \mathrm{AP}$ (SRLA1062; hueso humano) en el marco del análisis de los restos humanos encontrados en la Gruta de Candonga (Figura 1) a principios del siglo XX. Estos constituyen los restos humanos de mayor antigüedad datados hasta el momento en el territorio argentino (Cornero, Neves y Rivero, 2014).

Recientemente se identificó una ocupación discreta en un pequeño abrigo rocoso localizado en la pampa de Achala, denominado La Enramada 3 (en lo sucesivo LE3), en 


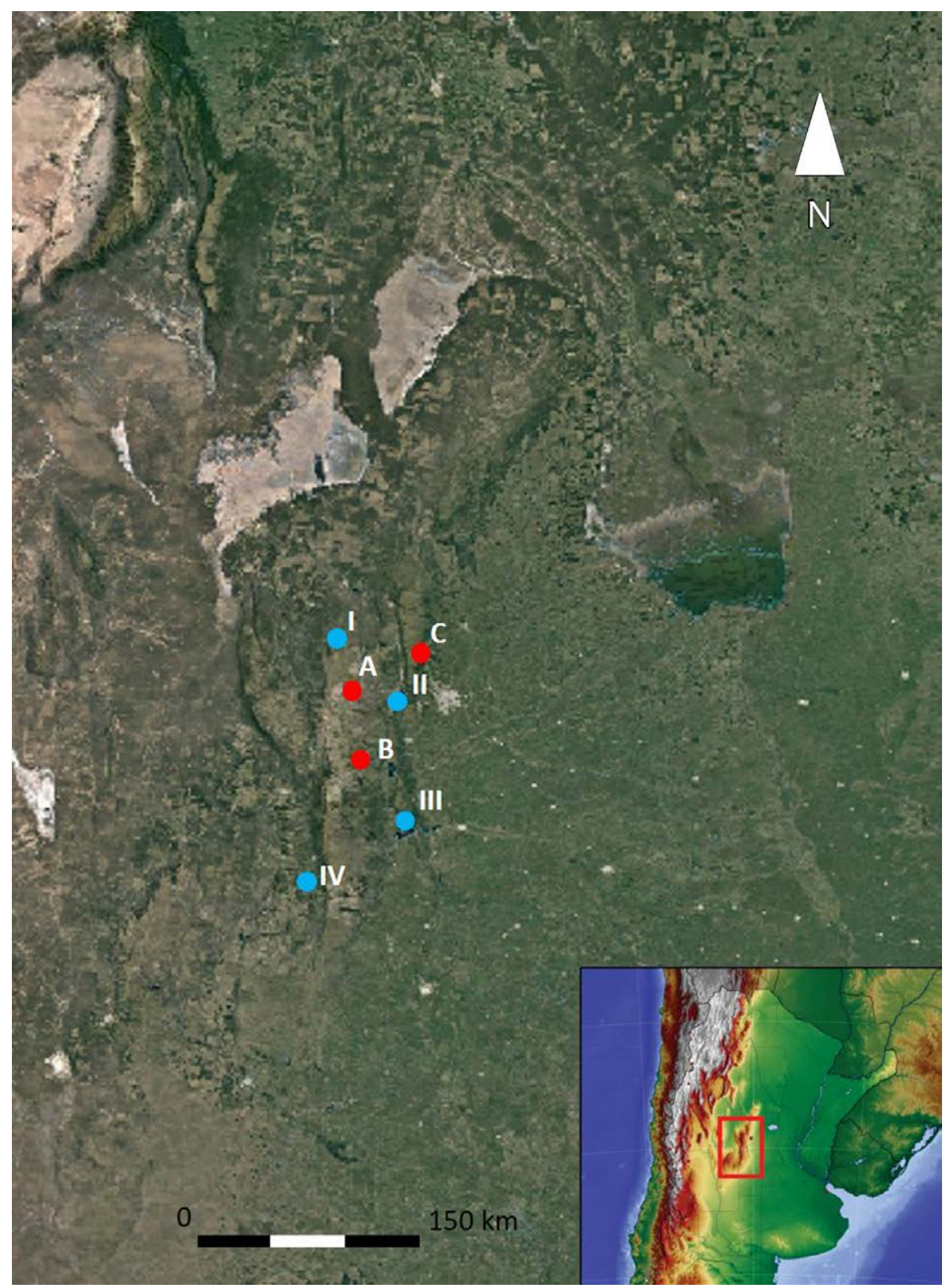

Figura 1. Sitios arqueológicos (Google Earth): A) El Alto 3; B) La Enramada 3; C) Gruta de Candonga. Hallazgos superficiales de puntas de proyectil PCP y Tigre: I) Characato; II) Lago San Roque; III) Embalse de Río Tercero; IV) Estancia la Suiza.

jurisdicción del Parque Nacional Quebrada del Condorito. La misma incluía artefactos, desechos líticos y restos de carbón, los cuales fueron datados en $10692 \pm 67$ años $\mathrm{C}^{14} \mathrm{AP}$ (D-AMS 025912). De este modo se consiguió datar un nuevo contexto arqueológico correspondiente a la transición Pleistoceno-Holoceno para el centro de Argentina.

Los registros en estratigrafía mencionados son complementados con la información proveniente de hallazgos superficiales de puntas de proyectil con diseños correspondientes a estos momentos tempranos; para la región de interés, "puntas cola de pescado" (PCP) y puntas “Tigre”. En este sentido, en la década de 1970 se produjo un hallazgo aislado de PCP en cercanías del Embalse de Río Tercero, en el Centro-Sur de la Sierra Grande de Córdoba (Schobinger, 1974). Investigaciones recientes en el sitio Estancia La Suiza (San Luis) permitieron identificar dos ejemplares fracturados 
(Laguens, Pautassi, Sario y Cattáneo, 2007). Asimismo, en las cercanías de Characato (Córdoba), se recolectó un pedúnculo de PCP (Cattáneo, Izeta y Caminoa, 2016). Finalmente, en las márgenes del Lago San Roque se localizaron una punta PCP (Rivero, Heider y Pastor, 2015) y una punta "Tigre" (Rivero, Pastor y Heider, 2018) (Figura 1). Las puntas PCP se han recuperado a lo largo de Sudamérica en numerosos sitios con fechas entre $c a .11000$ y 10000 años $C^{14} \mathrm{AP}$ (p. ej. Flegenheimer, Miotti y Mazzia, 2013; Loponte, Carbonera y Silvestre, 2015; Miotti y Terranova, 2015; Nami, 2007). Por su parte, las puntas "Tigre" constituyen un estilo de artefacto característico de los grupos tempranos que habitaron las planicies de Uruguay y sur de Brasil durante la transición Pleistoceno-Holoceno. Temporalmente pueden ser ubicadas entre ca. 10200 y 9700 años $\mathrm{C}^{14} \mathrm{AP}$ (p. ej. Suárez, 2016).

Las evidencias mencionadas son aún escasas en relación con otras regiones de Argentina y Sudamérica, a pesar de una tarea continua de más de 30 años de investigación arqueológica por parte de varios equipos multidisciplinarios (Cattáneo, Izeta y Costa, 2013). Recientemente se ha considerado que la baja densidad de sitios estaría indicando la existencia de una muy baja densidad poblacional durante este período, lo que pudo generar problemas para lograr una colonización y ocupación efectiva de la región (Rivero, 2012). En ese marco, se considera que las interrelaciones sociales debieron tener una importancia vital para el mantenimiento de la continuidad social y el éxito de los procesos de colonización inicial de una determinada región.

\section{Colonización de paisajes desconocidos y redes}

Para abordar las redes de interacción existentes entre las poblaciones de la región durante la transición Pleistoceno-Holoceno se siguieron los lineamientos del análisis de redes (Gamble, 2001). En este sentido, se analizaron las redes locales a través del estudio del transporte y uso de materias primas líticas, que constituyen indicadores apropiados de las dimensiones del paisaje de la costumbre (Gamble, 2001). Según este autor, es en ese espacio donde actúan las redes que posibilitan que cada persona persiga/logre sus fines reproductivos, económicos, políticos y sociales. Asimismo, a partir de la consideración del estilo de los artefactos que circularon entre las sociedades más tempranas, se definió el paisaje social (Gamble, 2001). Allí, por medio de una red ampliada, los individuos interactúan con un número mayor de personas, alejadas socialmente y/o espacialmente, gracias al uso de recursos simbólicos organizados por medio del estilo y la cultura material.

Rockman propone un modelo de aprendizaje del paisaje que aborda cómo los grupos humanos recolectan y comparten información ambiental desde la perspectiva de la colonización, planteando el rol del razonamiento, la percepción y la memoria en las interacciones humano-ambiente a través del tiempo (Rockman, 2003, 2009). El aprendizaje del paisaje se logra desarrollando el conocimiento ambiental, que se compone de tres partes: locacional, limitacional y social. El conocimiento locacional hace referencia a los recursos fijos e información relacionada geográficamente (p. ej. afloramientos rocosos, arroyos, distribución de aleros o cuevas, fuentes de materia prima, hábitats de presas). Este tipo de conocimiento puede ser recolectado y aprendido rápidamente, de acuerdo a las necesidades y afinidades del grupo. Por su parte, el conocimiento limitacional se refiere a las características cíclicas del ambiente y sus limitaciones para ciertos usos (p. ej. cantidad de precipitaciones, zonas inundables, capacidad de carga). Debido a que los ciclos de estos fenómenos ocurren a mediano plazo, no pueden ser aprendidos rápidamente y requieren un tiempo largo para ser incorporados en la memoria de los grupos. Finalmente, el conocimiento social describe un grupo de respuestas apropiadas al conocimiento locacional y limitacional, compartido y transmitido a las generaciones siguientes, formando un sentido colectivo de "así es como vivimos aquí" (Rockman, 
2009:54). Este último puede incorporar algún tipo de codificación del paisaje en forma de folklore, mapas cognitivos y toponímicos, lo que implica que este tipo de conocimiento requiere de un mayor tiempo (i.e. varias generaciones) para su desarrollo pleno (Kelly, 2003; Riesgo Chueca, 2010; Rockman, 2003, 2009). Es importante señalar que cada uno de estos tipos de conocimiento influye sobre los otros dos, por lo que cada uno de ellos puede ser actualizado en cualquier momento (Rockman, 2009).

A partir de los modelos de Gamble (2001) y Rockman (2009) es posible abordar arqueológicamente la exploración de nuevos territorios deshabitados, como es el caso del centro de Argentina durante el Tardiglacial. En el momento que se desprenden los grupos colonizadores del grupo mayor e ingresan en nuevos territorios, las redes locales y ampliadas influyeron en el modo en el cual los grupos exploraron/colonizaron el nuevo paisaje, pero a su vez sufrieron variaciones/adecuaciones conforme a las nuevas posibilidades y limitaciones que éste ofrecía (Figura 2).

Los grupos en su lugar de origen participan de una red local, con un acabado conocimiento locacional y limitacional del paisaje que habitan. Además, se encuentran incluidos en una red ampliada o paisaje social que permite el desarrollo del conocimiento social, producto del contacto con otros grupos más alejados espacialmente, con los que comparten un modo de vida y ciertos recursos simbólicos. Estas poblaciones, al desprenderse del grupo mayor e ingresar en un paisaje desconocido, realizan la exploración/conocimiento del mismo a partir de las preferencias y afinidades del conocimiento ambiental previo (Rockman, 2003). Es decir, la búsqueda de recursos fijos (p. ej. líticos, lugares para asentar campamentos, aleros) se realizará de acuerdo al conocimiento locacional y social que está incorporado en su memoria y que fue modelado en el lugar de origen. A medida que se van encontrando nuevos recursos o nuevas formas en las cuales éstos se presentan, el conocimiento locacional se va actualizando, al igual que el limitacional al pasar el tiempo. De ese modo se obtiene un mejor conocimiento del nuevo ambiente en proceso de colonización.

El conocimiento social, inicialmente, será el mismo que los grupos tienen incorporado desde su lugar de origen y, por su naturaleza, tardará más tiempo que los otros dos para actualizarse a las nuevas condiciones. Cuando este nuevo conocimiento social sea incorporado, el paisaje local ya no será "desconocido" y surgirá un nuevo paisaje social. El tiempo que transcurre entre el arribo al nuevo paisaje y su conocimiento efectivo es difícil de estimar y depende del grado de afinidad con el lugar de origen de los grupos, cuanto más afín sea el nuevo territorio más rápido se actualizará el conocimiento social (p. ej. Rockman, 2003, 2009).

En términos del modelo de aprendizaje del paisaje se espera que los grupos tengan incorporado el paisaje social de origen, con preferencias y formas de comportamiento adecuadas a este (i.e. conocimiento locacional, limitacional y social), y se vaya conformando la red local en base a estos conocimientos. La propuesta de Rockman (2009) puede ser evaluada arqueológicamente a través del estudio del uso y circulación de materias primas, así como desde el estilo de algunos tipos de artefactos. En este sentido, durante el poblamiento inicial, es decir los primeros exploradores que llegan a un nuevo territorio, tendrían sus toolkits compuestos en un importante porcentaje por materias primas de su lugar de origen, ya sea en forma de núcleos transportables o en artefactos conservados (p. ej. puntas de proyectil). Se espera que en el nuevo territorio inicialmente utilicen materias primas localmente disponibles, aunque no sean de muy buena aptitud para la talla, siguiendo una estrategia expeditiva (Franco, 2003, 2004; Nelson, 1991) hasta que logren detectar fuentes de materias primas de mejor calidad o que posean las características preferidas y/o buscadas. Esta etapa exploratoria es muy difícil de detectar arqueológicamente ya que se trataría de pequeños grupos muy dispersos que dejan una impronta material mínima (Borrero, 1994-95). 


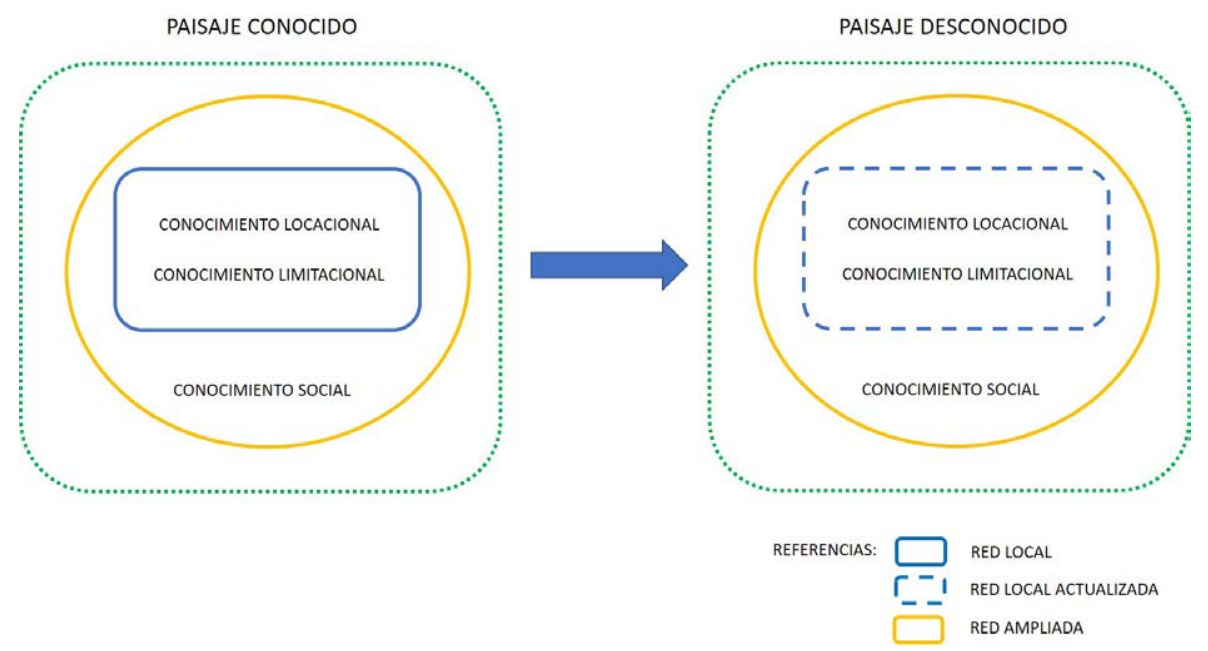

Figura 2. Modelo de exploración de paisajes desconocidos.

Una vez que varias generaciones de los grupos colonizadores han permanecido en el territorio, la red local se va "adaptando" al nuevo paisaje, actualizando el conocimiento locacional y limitacional, pero adecuándolos según el conocimiento social de origen. Esto podría ser detectado arqueológicamente por el uso de materias primas localizadas pero que se adaptan a las formas apropiadas para los grupos según el conocimiento social original, especialmente en el estilo de algunos artefactos, aunque se explotan nuevos recursos líticos que sean aptos para la talla en una forma regular y sistemática. Este proceso continuará hasta, eventualmente, conformar un nuevo paisaje social más adecuado al nuevo espacio ocupado.

\section{Base regional de recursos líticos}

Los estudios multidisciplinarios sobre materias primas líticas en la región arqueológica de Sierras Centrales (González, 1952) y sus llanuras adyacentes se han incrementado de manera notoria en los últimos años (p. ej. Cattáneo et al., 2018; Heider, Rivero y Baldo, 2015; Sario y Salvatore, 2018). En este contexto, se ha iniciado un proyecto multidisciplinario de escala macrorregional que abarca regiones llanas y semiáridas de las provincias de San Luis, La Rioja y Córdoba. El mismo tiene tres objetivos generales: a) detectar fuentes de materia prima y canteras arqueológicas; b) caracterizar ambas a nivel tecno-tipológico, petrográfico y geoquímico; c) analizar qué procesos geológicos y geomorfológicos intervinieron en su génesis y transformación. En ese marco espacial se propuso un primer modelo de yacencias de rocas silíceas de uso arqueológico para el centro de Argentina (Heider et al., 2018).

Los modos de yacencia se ordenaron en dos grupos principales a partir de la identificación de numerosas canteras arqueológicas y fuentes potenciales (Figura 3): A) las fuentes y canteras se ubicarían en rocas metamórficas constituyendo filones de cuarzo con dimensiones decimétricas o calcedonia en cuerpos irregulares de tamaños no superiores a $1 \mathrm{~m}$ ( $\mathrm{A}_{11}$ [El Chiquero, Borgo, Heider, Ramos y Curtoni, 2018]) o en venas discordantes o pequeños diques que cortan la foliación metamórfica de la roca de caja $\left(A_{12}[\right.$ La Suiza 2, Sario, 2013]). También en este grupo se reconoce calcedonia relacionada a granitos $\left(A_{2}\right)$, ya sea calcedonia en aplitas $\left(A_{21}\right.$ [Los Barrancos, Heider et al., 2018]), o cuarzo-calcedonia en cuerpos pegmatíticos ( $A_{22}$ [La Falla, Borgo et al., 2018]). Por otra parte, las calcedonias que se alojan en depósitos sedimentarios cenozoicos ricos en carbonatos (B), se pueden encontrar en bancos concordantes 


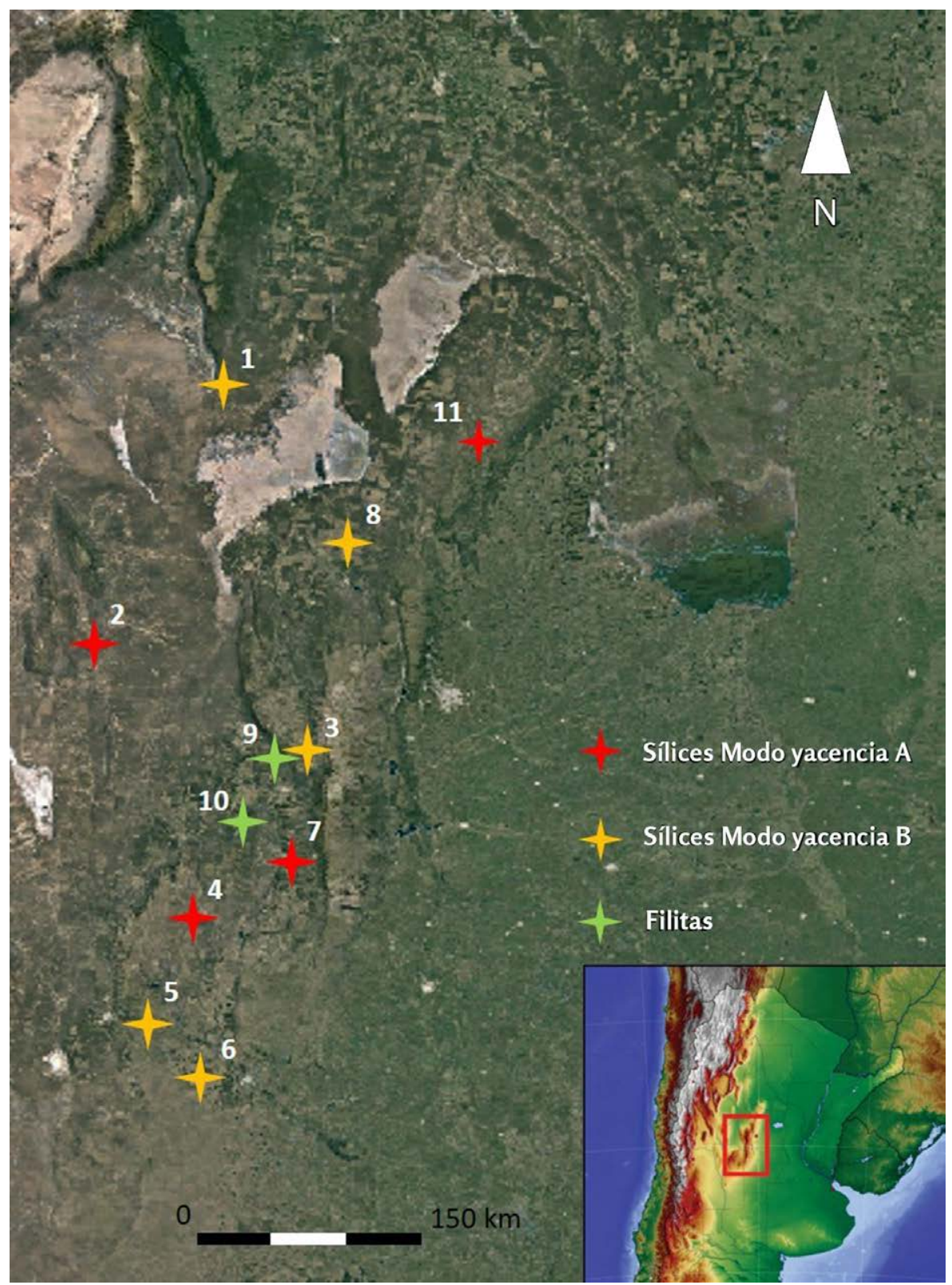

Figura 3. Fuentes de materias primas silíceas caracterizadas en el centro de Argentina (Google Earth): 1) Loma de Los Pedernales,;2) Los Barrancos; 3) Mina Clavero; 4) La Falla; 5) Paso de las Carretas; 6) El Lechuzo; 7) Estancia La Suiza; 8) El Ranchito-Copacabana; 9) Altautina; 10) Bajo de Véliz; 11) Sierra Norte.

con la estratificación horizontal o subhorizontal, con espesores superiores al metro (B ${ }_{1}$ [Lomas de Los Pedernales, Alto del Lechuzo, Mina Clavero, Heider et al., 2018; El Ranchito-Copacabana, Sario y Salvatore, 2018]) o en pequeñas concreciones que forman agregados irregulares ( $\mathrm{B}_{2}$ [Paso de Las Carretas, Borgo et al., 2018]).

\section{Uso de materias primas durante la transición Pleistoceno-Holoceno}

El uso de materias primas durante el Tardiglacial carece de antecedentes de investigación directo. Es una excepción a este punto el trabajo realizado por Sario (2011) con las fuentes de materia prima cercanas al sitio La Suiza (provincia de San Luis). Si bien 
1. Para el análisis lítico se siguieron las propuestas de Aschero (19751983). no pudo ser probado mediante fechados directos el uso temprano de las mismas, la identificación de dos fragmentos de PCP realizadas en la sílice local le permitió afirmar a la autora la utilización temprana de la misma. Sólo para momentos del Holoceno medio (8400 a 4200 AP) se ha abordado secundariamente el aprovisionamiento de sílices para la confección de puntas de proyectil lanceoladas (Heider y Rivero, 2018). En ese marco temporal, se ha propuesto un uso intenso de tres canteras de sílice (Loma de los Pedernales de Casa de Piedra (Catamarca), La Falla (San Luis) y El Ranchito (Córdoba) las cuales podrían haber sido desactivadas o escasamente utilizadas durante el Holoceno final (Borgo et al., 2018; Heider et al., 2018; Sario y Salvatore, 2018).

En este trabajo nos concentramos en los contextos tempranos recuperados en los sitios EA3 y LE3, constituidos casi exclusivamente por material lítico ${ }^{1}$. Estos sitios, con buen control estratigráfico, permitieron realizar una primera aproximación a las estrategias tecnológicas adoptadas y al uso de materias primas durante el período considerado. Es importante señalar que en ninguno de los dos se detectaron puntas de proyectil, entendidos como marcadores temporales indirectos ampliamente aceptados a nivel macrorregional.

El Componente 1A de EA3 incluye 397 desechos (Tabla 1, Figura 4). El 98,7\% (n = 392) son de cuarzo, siendo el resto de la muestra unidades aisladas de rocas identificadas como sílices (brecha, ópalo, calcedonia y sílice microcristalino) o rocas ígneas (pórfido). El análisis del origen de extracción (aún ante la dificultad de comparar número tan disímiles) dio como resultado que sólo una lasca de las rocas minoritarias fuera identificada como perteneciente a las primeras etapas de formatización de artefactos. En el caso del cuarzo, ese porcentaje representa casi el 12,5\%. En esta roca es notoria la ausencia de técnica bifacial para la manufactura de artefactos. Respecto al tamaño de las muestras recuperadas, se observa una concentración para todas las rocas en las lascas pequeñas o menores. Es una excepción para este patrón un pequeño porcentaje de lascas medianas y grandes para el cuarzo.

El Componente analizado en LE3 incluye 69 desechos (Tabla 2). El 95,6\% ( $n=66$ ) son de cuarzo, siendo el resto de la muestra tres elementos identificados como sílices microcristalinas. El análisis del origen de extracción permite observar que la totalidad de las sílices son lascas enteras o fracturadas con talón, mientras que en los cuarzos se incluyen casi un $37 \%$ de lascas fracturadas sin talón y elementos no diferenciados. En el cuarzo, casi el 25\% de las lascas identificadas pertenecen a las etapas iniciales de formatización, la cual no se observa en las sílices. Del mismo modo que sucede en EA3, los tamaños mayores sólo se ven representados en el cuarzo.

Los resultados del análisis de las materias primas empleadas en los artefactos retocados y los núcleos presentes muestran una preponderancia del cuarzo en ambos sitios (Tabla 3). Las rocas minoritarias están representadas por calcedonia $(n=2)$, pseudotaquilita $(\mathrm{n}=1)$ y ópalo $(\mathrm{n}=1)$ para EA3 y filita $(\mathrm{n}=1)$ y sílice $(\mathrm{n}=1)$ para LE3 (Figura 5). La mayor disponibilidad de cuarzo en el paisaje local se ve reflejada en la mayor variedad de grupos tipológicos de artefactos confeccionados en esa materia prima. Esto es notorio también en la presencia de núcleos no agotados en ambos sitios. En contraposición, las rocas minoritarias presentan núcleos en etapas más avanzadas de su vida útil. En este sentido, tanto el núcleo de sílice microcristalino como el de ópalo poseen características que posibilitan su transporte (i.e. tamaño mediano pequeño y pequeño) y fueron utilizados para obtener formas base de artefactos pequeños. El primero de ellos fue reducido mediante técnica bipolar y se encuentra agotado, lo que indica que se extendió su vida útil para obtener formas base adecuadas. Los instrumentos de rocas minoritarias en EA3 se pueden incluir en una idea de gestión tecnológica similar a los núcleos. Siendo estos de tamaño pequeño, confeccionados mediante retoques marginales (Tabla 3 ). 


\begin{tabular}{|c|c|c|c|}
\hline \multicolumn{4}{|c|}{ Fragmentación de la muestra } \\
\hline Materia prima & Cuarzo & Sílices & Ígneas \\
\hline NMD & $304(77,7 \%)$ & $4(100 \%)$ & $1(100 \%)$ \\
\hline LFST & $29(7,4 \%)$ & - & - \\
\hline INDI & $59(14,9 \%)$ & - & - \\
\hline Total & $392(100 \%)$ & $4(100 \%)$ & $1(100 \%)$ \\
\hline \multicolumn{4}{|c|}{ Origen de las extracciones } \\
\hline Materia prima & Cuarzo & Sílices & Ígneas \\
\hline Lascas externas & $38(12,5 \%)$ & $1(25 \%)$ & - \\
\hline Lascas internas & $252(82,9 \%)$ & $2(50 \%)$ & $1(100 \%)$ \\
\hline Lascas int. de formatiz. & $14(4,6 \%)$ & - & - \\
\hline Lascas de adelg. bif. & - & $1(25 \%)$ & - \\
\hline Total & $304(100 \%)$ & $4(100 \%)$ & $1(100 \%)$ \\
\hline \multicolumn{4}{|l|}{ Tamaño de los desechos } \\
\hline Materia prima & Cuarzo & Sílices & Ígneas \\
\hline Hipermicrolascas & $91(29,9 \%)$ & $1(25 \%)$ & - \\
\hline Microlascas & $156(51,3 \%)$ & $2(50 \%)$ & - \\
\hline Lascas pequeñas & $49(16,2 \%)$ & $1(25 \%)$ & $1(100 \%)$ \\
\hline Lascas & $5(1,6 \%)$ & - & - \\
\hline Lascas grandes & $3(1 \%)$ & - & - \\
\hline Total & $304(100 \%)$ & $4(100 \%)$ & $1(100 \%)$ \\
\hline
\end{tabular}

Tabla 1. Desechos líticos de EA3. Los porcentajes corresponden a los parciales y el total por cada materia prima que se presenta. Referencias: NMD) Número Mínimo de Desechos; LFST) Lascas Fracturadas Sin Talón; INDI) Desechos Indiferenciados.

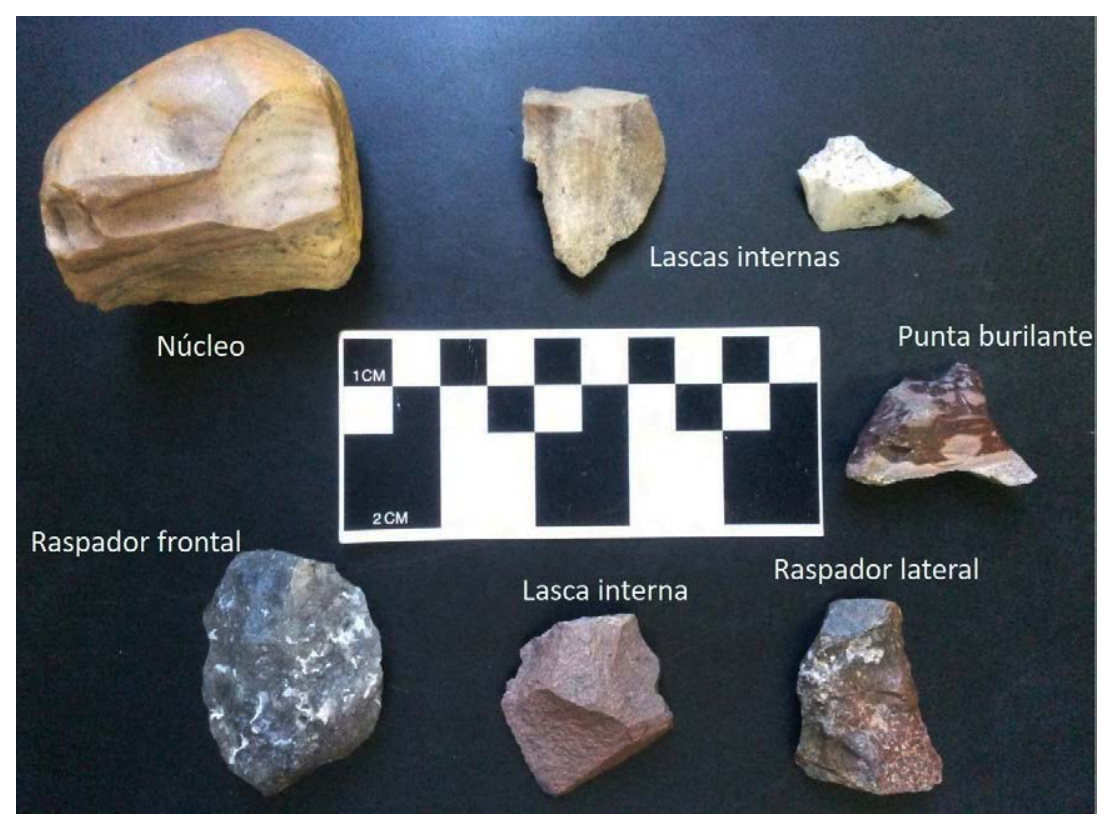

Figura 4. EA3. Artefactos y núcleos de materias primas silíceas distintas al cuarzo. 


\begin{tabular}{|c|c|c|c|}
\hline \multicolumn{4}{|c|}{ Fragmentación de la muestra } \\
\hline Materia prima & Cuarzo & Sílices & Ígneas \\
\hline NMD & $42(63,6 \%)$ & $3(100 \%)$ & - \\
\hline LFST & $19(28,8 \%)$ & - & - \\
\hline INDI & $5(7,6 \%)$ & - & - \\
\hline Total & $66(100 \%)$ & $3(100 \%)$ & - \\
\hline \multicolumn{4}{|c|}{ Origen de las extracciones } \\
\hline Materia prima & Cuarzo & Sílices & Ígneas \\
\hline Lascas externas & $11(26,2 \%)$ & - & - \\
\hline Lascas internas & $24(57,1 \%)$ & $2(66,6 \%)$ & - \\
\hline Lascas int. de formatiz. & $6(14,2 \%)$ & - & - \\
\hline Lascas de adelg. bif. & $1(2,5 \%)$ & $1(33,4 \%)$ & - \\
\hline Total & $42(100 \%)$ & $3(100 \%)$ & - \\
\hline \multicolumn{4}{|l|}{ Tamaño de los desechos } \\
\hline Materia prima & Cuarzo & Sílices & Ígneas \\
\hline Hipermicrolascas & $11(26,2 \%)$ & - & - \\
\hline Microlascas & $18(42,9 \%)$ & $2(66,6 \%)$ & - \\
\hline Lascas pequeñas & $7(16,6 \%)$ & $1(33,4 \%)$ & - \\
\hline Lascas & $4(9,5 \%)$ & - & - \\
\hline Lascas grandes & $2(4,8 \%)$ & - & - \\
\hline Total & $42(100 \%)$ & $3(100 \%)$ & - \\
\hline
\end{tabular}

Tabla 2. Desechos líticos de LE3. Los porcentajes corresponden a los parciales y el total por cada materia prima que se presenta. Referencias: NMD) Número Mínimo de Desechos; LFST) Lascas Fracturadas Sin Talón; INDI) Desechos Indiferenciados.

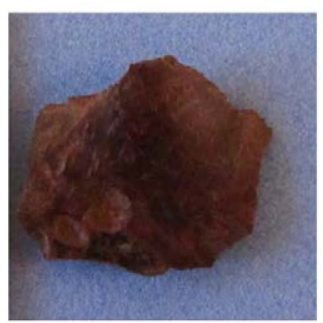

Lasca de adelgazamiento bifacial

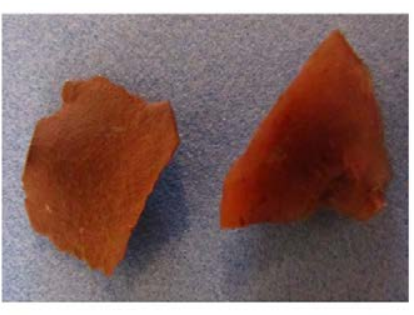

Lascas de formatización
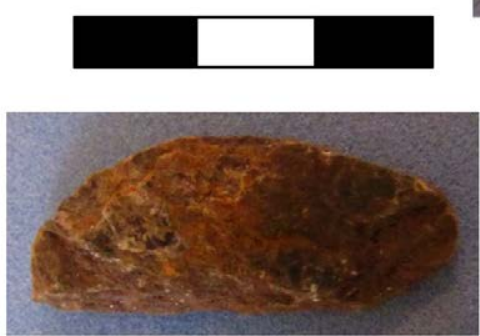

Artefacto de filita

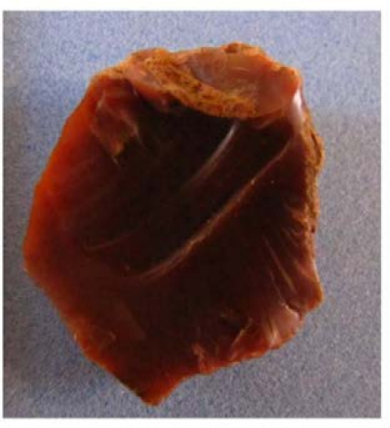

Núcleo bipolar

Figura 5. LE3. Artefactos y núcleos de materias primas distintas al cuarzo. 


\begin{tabular}{|c|c|c|c|}
\hline \multicolumn{4}{|c|}{ El Alto 3} \\
\hline Grupos Tipológicos & $\mathbf{n}$ & $\%$ & Materia prima \\
\hline Raspador & 2 & 11,8 & Cuarzo $(n=1)$, Calcedonia $(n=1)$ \\
\hline Artefacto sumario indiferenciado & 1 & 5,9 & Cuarzo \\
\hline Artefacto sumario filo marginal corto & 2 & 11,8 & Cuarzo, Calcedonia \\
\hline Artefacto sumario filo ultramarginal largo & 1 & 5,9 & Cuarzo \\
\hline Punta burilante & 2 & 11,8 & Cuarzo $(n=1)$, Pseudotaquilita $(n=1)$ \\
\hline Percutor & 1 & 5,9 & Cuarzo \\
\hline Muesca de lascado simple & 1 & 5,9 & Cuarzo \\
\hline Muesca retocada & 1 & 5,9 & Cuarzo \\
\hline Artefacto compuesto (raspador + muesca) & 2 & 11,8 & Cuarzo \\
\hline Núcleo & 4 & 23,3 & Cuarzo ( $(n=3)$, Ópalo $(n=1)$ \\
\hline Total & 17 & 100 & - \\
\hline \multicolumn{4}{|c|}{ La Enramada 3} \\
\hline Grupos Tipológicos & $\mathrm{nN}$ & $\%$ & Materia prima \\
\hline Raspador & 2 & 40 & Cuarzo \\
\hline Placa (fragmento) & 1 & 20 & Filita \\
\hline Núcleo & 2 & 40 & Cuarzo $(n=1)$, Sílice $(n=1)$ \\
\hline Total & 5 & 100 & - \\
\hline
\end{tabular}

Tabla 3. Artefactos de $E_{3}$ y $L E_{3}$.

Un análisis integral de las rocas identificadas permite observar que la predominancia notoria del cuarzo en los desechos de talla (97\%) se reduce al analizar los artefactos formatizados y núcleos (88\%). Esto sugiere que los artefactos de rocas minoritarias ingresaban al sitio en etapas avanzadas de su vida útil, conformando una estrategia de conservación (Nelson, 1991). Por su parte, los artefactos de cuarzo fueron manufacturados y empleados en una forma más acorde a las estrategias expeditivas (Nelson, 1991) o utilitarias (Escola, 2001), siendo descartados en los sitios con los filos aún activos.

Finalmente, los hallazgos superficiales de puntas de proyectil PCP y Tigre también aportan información útil para la discusión sobre el uso de materias primas durante el poblamiento temprano de la región (Figura 6). En este sentido, la gestión tecnológica de todos los cabezales líticos incluyó adelgazamiento bifacial sobre lascas de rocas silíceas. Esta técnica fue identificada en instrumentos y desechos de talla en las rocas minoritarias tanto de EA 3 como LE3, siendo mínima la presencia de esta forma de reducción en el cuarzo. Las posibles fuentes de procedencia de las rocas minoritarias son acotadas espacialmente respecto a la abundancia del cuarzo, aún si fueran propias de la región como es el caso de La Suiza 2 (Heider et. al., 2018; Sario, 2013). De este modo la estrategia de conservación constituiría una forma de gestión adecuada para el manejo de un recurso de alta calidad, en un paisaje sólo parcialmente conocido.

\section{Discusión}

El registro arqueológico proveniente de los contextos datados en el tardiglacial en la Sierras Centrales (EA3 y LE3) está constituido casi exclusivamente por material lítico. Los conjuntos presentados están compuestos mayoritariamente por cuarzo, con un 

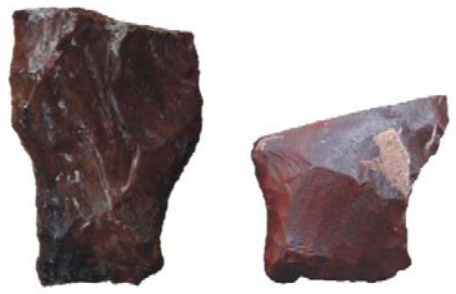

Estancia La Suiza 2

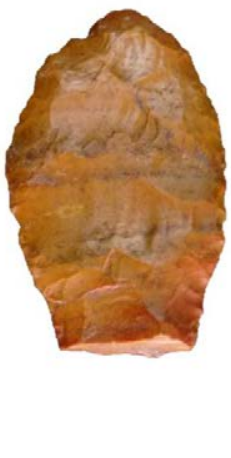

Lago San Roque

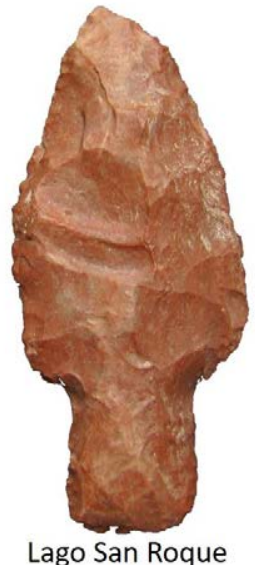

Lago San Roque

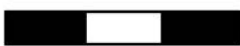

Figura 6. Puntas de proyectil PCP (izquierda y centro) y Tigre (derecha) y sus lugares de hallazgo.

mínimo porcentaje de otras materias primas correspondientes, fundamentalmente, a distintas variedades de sílice. El cuarzo es una roca de regulares a buenas aptitudes para la talla y está disponible ampliamente en el área (Cattáneo et al., 2018; Heider et al., 2018). Estas dos características (amplia disponibilidad y condiciones aptas para la talla), se adaptarían a las necesidades de obtener una importante variedad de artefactos de manera expeditiva (filos, raspadores, raederas, puntas burilantes, entre otros) para las tareas consuetudinarias. En este sentido, la presencia de las fuentes en el entorno inmediato de los sitios permitiría a los grupos colonizadores apropiarse del recurso en forma rápida, sin implicar costosas exploraciones buscando otras fuentes de rocas. Apoyan esta idea las tendencias en la gestión tecnológica identificada: altos porcentajes de cuarzo verificados en los sitios, presencia de núcleos no agotados, artefactos descartados en etapas tempranas de su vida útil y lascas de todas las etapas de confección de artefactos.

De forma opuesta, los grupos colonizadores emplearon una lógica de gestión tecnológica diferente para las rocas sílices. Creemos que su carácter minoritario en los sitios tempranos no responde a un proceso de formación de sitio, sino a una cuestión social. Tanto las lascas recuperadas (todas de etapas intermedias o avanzadas del proceso de manufactura), como así también los instrumentos y núcleos, muestran la presencia de secuencias de manufactura prolongadas en el tiempo y esfuerzos en la reactivación de los artefactos, coherentes con una tecnología que podríamos considerar conservada (Nelson, 1991). La identificación de las fuentes de sílices se encuentra en desarrollo (Heider et al., 2015, 2018), siendo Estancia la Suiza 2 la única con evidencias de uso para momentos tempranos (Sario, 2013). Desde el estudio litológico del material de los sitios sólo se ha avanzado en la caracterización de un espécimen (i.e. una lasca interna proveniente de EA3) que fue determinado como calcedonia mediante cortes petrográficos y DRX, aunque no pudo vincularse a ninguna fuente conocida. En cuanto a la filita recuperadas en LE3, no se registran estudios sobre las fuentes, aunque las mismas fueron posicionadas desde investigaciones bibliográficas y geológicas en Altautina (oeste de las Sierras de Córdoba; Heider et al., 2015) y en Bajo de Véliz (noreste de la Sierra de San Luis; Heider et al., 2015) (Figura 4).

Como se mencionó, el análisis de los modos de yacencia (Heider et al., 2018) permitió definir dos tipos principales. Todas las fuentes y canteras propias del grupo B (aquellas que alojan en depósitos sedimentarios cenozoicos ricos en carbonatos) 
tienen características macroscópicas similares en cuanto a: a) color (en su mayoría gris nube con tonos blancos lechosos concentrado en los bordes externos); b) forma de presentación (concreciones nodulares, subesféricas o elípticas y botroidales, con tamaños máximos registrados de $32 \mathrm{~cm}$ de diámetro); c) impurezas (huecos vacíos o microgeodes); d) disponibilidad en áreas de llanuras y sectores peri-salinares (Loma de los Pedernales de Casa de Piedra en Catamarca y Esquina del Norte en La Rioja), barrancas sometidas a procesos erosivos en espacios peri-serranos (Mina Clavero, Córdoba) y llanuras de clima semiárido (Alto del Lechuzo y Loma de Los Pedernales en San Luis). Ninguna de las sílices recuperadas en EA3 y LE3 tienen estas características. Por el contrario, por su forma, color y corteza parecieran responder más a las características del "Modo de Yacencia A". En ese sentido, las únicas dos piezas arqueológicas caracterizadas temporalmente como tempranas, y con fuente de procedencia conocidas, son la PCP de brechas silíceas procedentes de La Suiza 2 (Sario, 2011). Esta localidad arqueológica fue utilizada como "fuente tipo" para caracterizar el "Modo de Yacencia $\mathrm{A}_{12}$ " de rocas silíceas en foliación metamórfica de la roca de caja.

Si se analiza el aprovisionamiento de los conjuntos líticos estratigráficos asignados a la transición Pleistoceno-Holoceno desde el punto de vista de la red local (Gamble, 2001) y los modelos de aprendizaje (Rockman, 2003, 2009) se observa un uso diferente y tal vez complementario de las rocas. Entendemos que el uso del cuarzo pudo satisfacer las necesidades de obtener artefactos de manera inmediata debido a su ubicación y características técnicas (recurso fijo y altamente disponible con baja incertidumbre espacio-temporal y buenas aptitudes para la talla); por ello, su localización debió ser rápidamente incorporada al conocimiento locacional de los grupos. Por su parte, las rocas minoritarias, debido a la naturaleza de su localización muy puntual en el espacio, seguramente requirieron mayor tiempo de exploración para ser incorporadas al conocimiento locacional. En este sentido, las sílices se emplearon para la manufactura de instrumentos transportables, dentro de una estrategia de conservación (i.e. puntas de proyectil, raspadores, etc.), que fueron descartados al estar agotados sus filos o fracturados en el caso de las puntas PCP. Esto indica que estas materias primas eran importantes para esos grupos más allá de sus aptitudes para la talla. En este sentido, la calidad del cuarzo permitía también la confección de PCP o bifaces. Sin embargo, esos artefactos no fueron confeccionados sino con sílices de colores, por cuanto la calidad no fue un elemento excluyente en su selección. Es posible que su elección esté basada en preferencias de índole social/simbólica, como se ha postulado para casos de la región de Tandilia (Flegenheimer y Bayón, 1999). En el mismo sentido, esa selección estaría ligada al mantenimiento de redes sociales amplias, con grupos alejados espacialmente desde donde se originó el vector inicial de ocupación y cuyo paisaje social se busca reproducir (Gamble, 2001).

La ubicación espacial de las posibles fuentes sería también un indicador de interés. Siguiendo a Rockman (2003, 2009), el conocimiento social de los grupos colonizadores guardaría una información ambiental, una percepción, una memoria y los razonamientos consecuentes con la ubicación de las fuentes en la región de origen del grupo. Si el poblamiento de Sierras Centrales tuvo un fuerte vector esteoeste (Fabra, 2014; Laguens, 2006), los grupos colonizadores habrían contado con un conjunto de conocimientos que les permitieron explotar con mayor facilidad rocas de buena calidad para la talla (y con una variedad de colores propuesta como elemento de valor simbólico) en puntos elevados del paisaje (donde se encuentran las sílices del Modo de Yacencia A). En contraposición, es poco probable que desde dicho conocimiento social se haya podido acceder a diferentes espacios peri-serranos llanos y semidesérticos (donde se encuentran las sílices del Modo de Yacencia B) que tenían condiciones ambientales muy diferentes al que poseía como background el grupo colonizador (Figura 7). 


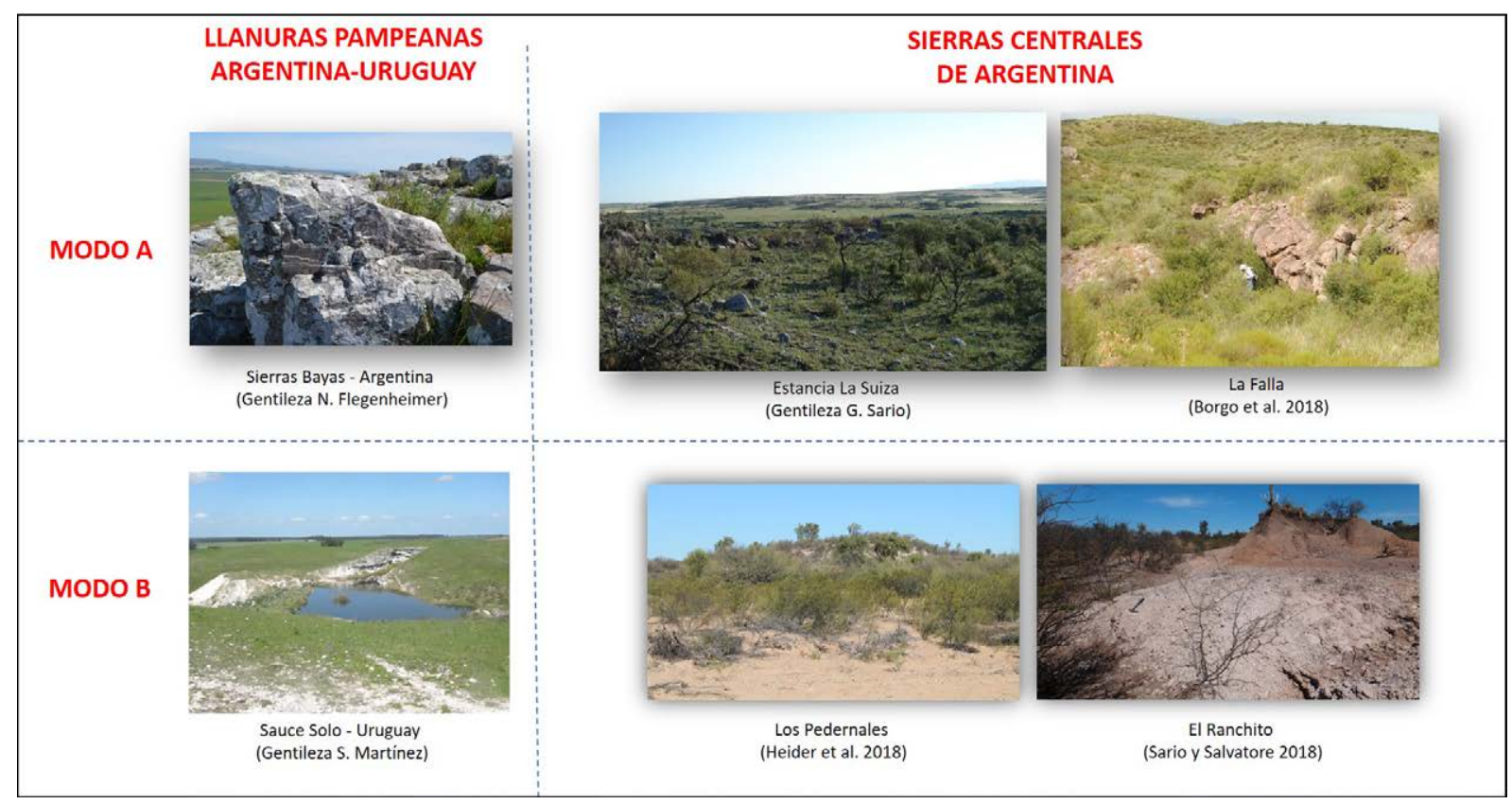

Figura 7. Comparación de Modos de Yacencia similares en las llanuras pampeanas de Argentina y Uruguay.

Finalmente, la identificación de una punta de proyectil "Tigre" en las Sierras Centrales permitió proponer algún tipo de contacto con los grupos del sector noroeste de Uruguay (Rivero et al., 2018). En esa región las fuentes de materia prima son identificadas como calizas del Queguay, similar a las fuentes del modo B del centro de Argentina (Heider et al., 2018; Martinez, Veroslavsky y Cabrera, 2015). Este caso constituye aún un hallazgo aislado como para pensar un proceso de colonización importante desde ese sector, el cual podría compartir conocimiento social necesario para identificar las fuentes de calcedonias traslucidas en los llanos semidesérticos peri-serranos. Adicionalmente se debe señalar que esta tipología de puntas es tardía respecto a las PCP (p. ej. Suárez, 2016). Su presencia en torno a $10200 \mathrm{AP}$ se encuentra más cercana en el tiempo al hiato ocupacional de finales del Holoceno temprano, el cual es entendido como una consecuencia de la extinción local o restricción de la población serrana (Rivero, 2012). En este sentido, se ha propuesto como una de las posibles causas de este fenómeno al distanciamiento del grupo de origen, lo que impactaría sobre la viabilidad genética de la población, si esta es muy pequeña, como indicarían las reducidas evidencias arqueológicas obtenidas.

\section{Conclusión}

Durante la transición Pleistoceno-Holoceno las poblaciones serranas compartían preferencias y "maneras de hacer las cosas" que guiaron la búsqueda y el empleo de las materias primas líticas. Estas formaban parte del conocimiento social que tenían incorporado y que compartían con aquellos grupos del lugar de origen. A medida que el conocimiento locacional y limitacional se actualizaba y se establecían nuevas redes locales, también se habría actualizado el conocimiento social, adaptándose al nuevo ambiente. Sin embargo, la red ampliada o paisaje social se habría mantenido, vinculando a los grupos serranos con su lugar de origen, como lo sugiere la pervivencia de los diseños de puntas de proyectil PCP y las maneras de explotar y manipular las materias primas silíceas (Laguens, 2012). Esto habría actuado como un reaseguro para la viabilidad de las poblaciones colonizadoras, manteniendo lazos (reales o potenciales) con los núcleos de población de los cuales provenían, que resultarían esenciales si 
tenemos en cuenta la bajísima densidad poblacional en el área central de Argentina, sugerida por las escasas evidencias y dataciones disponibles (p. ej. Rivero, 2012).

Si se considera válida esta propuesta de aplicación del modelo de redes y exploración del paisaje a partir de la contrastación de nuevas evidencias estratigráficas, será posible explorar en el futuro su validez para otros momentos de la ocupación del centro de Argentina. En este sentido, si el paisaje social de los primeros pobladores estuvo relacionado con un vector de ingreso desde el oriente, el cual se pierde (al menos en el registro arqueológico) hacia finales del Holoceno temprano (Rivero, 2012), es posible proponer que la tecnología "Ayampitin" (característica del Holoceno medio en la región) esté relacionada con otra población que participa de otro paisaje social, propio de la región andina central de Argentina.

\section{Agradecimientos}

Deseamos agradecer a Edgardo Baldo, Sebastián Pastor y Ariel Ortiz Suarez por sus discusiones acerca de la identificación de fuentes de sílices y su empleo prehispánico. Colaboraron generosamente con algunas de las imágenes Nora Flegenheimer, Gisela Sario, Sergio Martínez y Rafael Curtoni. Esta investigación se financió con el apoyo de los subsidios Secyt-UNC 33620180100816CB y CONICET (PIP 11220080102678). Agradecemos a los evaluadores anónimos que contribuyeron a mejorar el manuscrito. 


\section{Q Referencias citadas}

"Ameghino, F. (1885). Informe del Museo Antropológico y Paleontológico de la Universidad Nacional de Córdoba durante el año 1885. BANC, 5, 257-306.

" Aschero, C. A. (1975). Ensayo para una clasificación morfológica de artefactos líticos aplicada a estudios tipológicos comparativos. Informe al CONICET. Manuscrito inédito.

" Aschero, C. A. (1983). Registro de códigos para atributos descriptivos aplicados a artefactos líticos. Informe al CONICET. Manuscrito inédito.

» Borgo, M., Heider, G., Ramos G. y Curtoni, R. (2018). Primeros avances en el área de canteras La Falla (provincia de San Luis). En R. Cattáneo, A. Izeta, T. Costa y G. Sario (Eds.), I Congreso Argentino de Estudios Líticos en Arqueología (pp. 32-34). Córdoba: Universidad Nacional de Córdoba.

»Borrero, L. (1994-95). Arqueología de la Patagonia. Palimpsesto. Revista de Arqueología, 4, 9-69.

»Castellanos, A. (1943). Antigüedad geológica de los restos humanos de la Gruta de Candonga. Publicaciones del Instituto de Fisiografía y Geología. Universidad Nacional del Litoral, XIV.

" Cattáneo, R., Izeta, A. y Caminoa, J. (2016). A Fishtail Projectile Point from the Southern Pampean Hills, Characato, Córdoba, Argentina, PaleoAmerica, 2(3), 274-276.

" Cattáneo, R., Izeta, A. y Costa T. (2013). El patrimonio arqueológico de los espacios rurales de la provincia de Córdoba. Córdoba: Universidad Nacional de Córdoba.

»Cattáneo, R., Sario, G., Caminoa, J., Collo, G., Rubio, M., Germanier, A., Faudone, S., Izeta, A. y Salvatore, M. (2018). Caracterización química de fuentes de abastecimiento e instrumental arqueológico de cuarzo de la provincia de Córdoba (Argentina). Trabajo presentado en las VII Jornadas Arqueológicas de Cuyo, Malargüe, Argentina.

»Cornero, S., Neves, W. y Rivero, D. (2014). Nuevos aportes a la cronología de las ocupaciones tempranas en las sierras de Córdoba. La Gruta de Candonga (Córdoba, Argentina). Relaciones de la Sociedad Argentina de Antropología, XXXIX, 285-292.

"Escola, P. (2001). Tecnología Lítica y Sociedades Agro-pastoriles Tempranas. (Tesis Doctoral inédita), Facultad de Filosofía y Letras, Universidad de Buenos Aires, Argentina.

" Fabra, M. (2014). Historia de las poblaciones prehispánicas del sector austral de las sierras pampeanas: variabilidad morfológica y modelos arqueológicos. Córdoba: Editorial de la Facultad de Filosofía y Humanidades, Universidad Nacional de Córdoba.

"Flegenheimer, N. y Bayón, C. (1999). Abastecimiento de Rocas en Sitios Pampeanos Tempranos: Recolectando Colores. En C. Aschero, A. Korstanje y P. Vuoto, P. (Eds.), Los Tres Reinos, Prácticas de Recolección en el Cono Sur de América (pp. 95-107). Tucumán: Ediciones Magna Publicaciones.

» Flegenheimer, N., Miotti, L. y Mazzia, N. (2013). Rethinking early objects and landscapes in the Southern Cone: Fishtail-point concentrations in the Pampas and Northern Patagonia. En K. Graf, C. Ketron y M. Waters (Eds.), Paleoamerican Odyssey (pp. 359376). Texas: College Station, Center for the Study of the First Americans, Texas A\&M University.

》Franco, N. (2003). ¿Es posible diferenciar los conjuntos líticos atribuidos a la exploración de un espacio de los correspondientes a otras etapas del poblamiento? El caso del extremo sur de Patagonia. Revista Werken, 3, 119-132. 
»Franco, N. (2004). La organización tecnológica y el uso de escalas espaciales amplias. El caso del sur y oeste de Lago Argentino. En A. Acosta, D. Loponte y M. Ramos (Comps.), Temas de Arqueología. Análisis Lítico (pp. 101-144). Luján: Universidad Nacional de Luján.

»Gamble, C. (2001). Las sociedades paleolíticas de Europa. Barcelona: Ariel.

»González, A. (1952). Antiguo horizonte precerámico en las Sierras Centrales de la Argentina. Runa, V, 110-133.

» González, A. (1960). La estratigrafía de la Gruta de Intihuasi (Prov. de San Luis, R.A.) y sus relaciones con otros sitios precerámicos de Sudamérica. Revista del Instituto de Antropología, 1, 5-296.

» Heider, G., Ortiz Suarez, A., Rivero, D., Baldo, E., Pastor, S., Ramos, G., Borgo, M., Gil, R., Chiesa, J., Costa, C., Recalde, A., Curtoni, R., Capriolo, J. y Muñoz, L. (2018). Estudios geoarqueológicos multiproxy de fuentes y canteras líticas de las Sierras Pampeanas y Ilanuras adyacentes. En R. Cattáneo, A. Izeta, T. Costa y G. Sario (Eds.), I Congreso Argentino de Estudios Líticos en Arqueología (pp. 69-70). Córdoba: Universidad Nacional de Córdoba.

» Heider, G. y Rivero, D. (2018). Estudios morfométricos aplicados a puntas de proyectil lanceoladas “Ayampitín” del Holoceno Temprano-medio en las Sierras y Llanuras Pampeanas Argentinas, Sudamérica. Latin American Antiquity, 29(3), 572-590.

» Heider G., Rivero, D. y Baldo, E. (2015). Rocas de uso arqueológico en Sierras Centrales. Fuentes de recursos líticos identificadas y potenciales en las provincias de Córdoba y San Luis, Argentina. Revista de Antropología del Museo de Entre Ríos, 1(2), 55-72.

» Kelly, R. (2003). Colonization of New land by Hunter-gatherers. Expectations and implications based on ethnographic data. En M. Rockman y J. Steele (Eds.), Colonization of Unfamiliar Landscapes: The Archaeology of Adaptation (pp. 44-58). Londres: Routledge.

»Laguens, A. (2006). El poblamiento inicial del sector austral de las Sierras Pampeanas de Argentina desde la Ecología del Paisaje. Revista Anales de Arqueología y Etnología de la Facultad de Filosofía y Letras de la Universidad Nacional de Cuyo, 62, 67-106.

»Laguens, A. (2012). La rutinización de las prácticas materiales, la memoria social y la cimentación del habitar en el devenir del poblamiento inicial del centro de Argentina. Trabajo presentado en el VI Simposio Internacional: El Hombre Temprano en América, Colombia.

»Laguens, A., Pautassi, E., Sario, G. y Cattáneo, R. (2007). Fishtail Projectile Points from Central Argentine. Current Research in the Pleistocene, 24, 55-57.

» Loponte, D., Carbonera, M. y Silvestre, R. (2015). Fishtail Projectile Points from South America: The Brazilian Record. Archaeological Discovery, 3, 85-103.

» Martinez, S., Veroslavsky, G. y Cabrera, F. (2015). Calizas del Queguay: Un enfoque hacia la arqueología. Revista de Antropología del Museo de Entre Ríos, 1(2), 1-10.

» Miotti, L. y Terranova, E. (2015). A Hill Full of Points in Terra Incognita from Patagonia: Notes and Reflections for Discussing the Way and Tempo of Initial Peopling. PaleoAmerica, 1(2), 181-196.

» Nami, H. (2007). Research in the Middle Negro River Basin (Uruguay) and the Paleoindian Occupation of the Southern Cone. Current Anthropology, 48, 164-174.

» Nelson, M. (1991). The Study of Technological Organization. En M. Schiffer (Ed.), Archaeological Method and Theory Vol. 2 (pp. 57-100). Tucson: University of Arizona Press.

» Riesco Chueca, P. (2010). Nombres en el paisaje: la toponimia, fuente de conocimiento y aprecio del territorio. Cuadernos Geográficos, 46(1), 7-34.

» Rivero, D. (2009). Ecología de cazadores-recolectores del sector central de las Sierras de Córdoba (Rep. Argentina). Oxford: BAR International Series 2007. 
》 Rivero, D. (2012). La ocupación humana durante la transición Pleistoceno-Holoceno (11,000 - 9,000 a.P.) en las Sierras Centrales de Argentina. Latin American Antiquity, 23(4), 551-564.

» Rivero, D. y Berberián, E. (2006). El poblamiento inicial de las Sierras Centrales de Argentina. Las evidencias arqueológicas tempranas. Cazadores Recolectores del Cono Sur. Revista de Arqueología, 1, 127-138.

» Rivero, D. y Berberián, E. (2008). El poblamiento de la región central del territorio argentino durante la Transición Pleistoceno-Holoceno (1200o - 9000 A.P.). Revista Española de Antropología Americana, 38(2), 17-37.

» Rivero, D., Heider, G. y Pastor, S. (2015). Identificación de una punta cola de pescado en las Sierras Centrales, implicancias para un modelo de poblamiento del centro de Argentina. Cuadernos del Instituto de Antrpología y Pensamiento Latinoamericano, 24(1), 151-155.

» Rivero, D., Pastor S. y Heider, G. (2018). The Tigre Projectile Point in Central Argentina: Implications for the Initial Peopling of the Region, PaleoAmerica, 41(1), 68-70.

» Rivero, D. y Roldán, F. (2005). Initial Peopling of the Cordoba Mountains, Argentina: First Evidence from El Alto 3. Current Research in the Pleistocene, 22, 33-34.

» Rockman, M. (2003). Knowledge and Learning in the Archaeology of Colonization. En M. Rockman y J. Steele (Eds.), Colonization of Unfamiliar Landscapes: The Archaeology of Adaptation (pp. 3-24). Londres: Routledge.

» Rockman, M. (2009). Landscape Learning in Relation to Evolutionary Theory. En A. M. Prentiss, I. Kuijt y J. C. Chatters (Eds.), Macroevolution in Human Prehistory (pp.51-71). New York: Springer.

» Roldán, F., Rivero, D. y Pastor, S. (2005). Las Sierras Centrales durante el Holoceno: perspectivas desde El Alto III (Pampa de Achala, Provincia de Córdoba). En E. Pillado (Ed.), Actas del XIII Congreso Nacional de Arqueología Argentina, Tomo 4 (pp. 277-286). Córdoba: Brujas.

"Sario, G. (2011). Poblamiento humano en la provincia de San Luis: una perspectiva arqueológica a través del caso de la organización de la tecnología en Estancia La Suiza. (Tesis Doctoral inédita), Facultad de Filosofía y Humanidades, Universidad Nacional de Córdoba, Argentina.

»Sario, G. (2013). Sources of lithic material procurement in Estancia La Suiza archeological locality (San Luis, Argentina). Journal of Archaeological and Anthropological Sciences, 5(3), 245-254.

»Sario, G. y Salvatore, M. (2018). Caracterización petrográfica y disponibilidad de recursos líticos en la cuenca del río Copacabana, noroeste de Córdoba, Argentina. Mundo De Antes, 12(2), 43-66.

"Schobinger, J. (1974). Nuevos hallazgos de puntas “Cola de Pescado" y consideraciones en torno al origen y dispersión de la Cultura de Cazadores Superiores Toldense en Sudamérica. En V. Cerulli (Ed.), Atti del XL Congreso Internazionale degle Americanisti (pp. 33-50). Roma-Genova: Tilgher.

»Serrano, A. (1945). Los Comechingones. Serie Aborígenes Argentinos I. Córdoba: Instituto de Arqueología, Lingüística y Folcklore de la Universidad Nacional de Córdoba.

»Suárez, R. (2016). The human colonization of the Southeast Plains of South America: Climatic conditions, technological innovations and the peopling of Uruguay and south of Brazil. Quaternary International, 431, 181-193.

»Vignati, M. (1940). Culturas prehistóricas y protohistóricas de la provincia de San Luis. Antecedentes bibliográficos: los precursores. Notas del Museo de La Plata - Antropología, 20(V), 149-176. 